\title{
The Negative Impact of Vehicular Intelligence on Energy Consumption
}

\author{
Zongwei Liu, ${ }^{1,2}$ Hong Tan, ${ }^{1,2}$ Xu Kuang, ${ }^{1,2}$ Han Hao, ${ }^{1,2}$ and Fuquan Zhao $\mathbb{D}{ }^{1,2}$ \\ ${ }^{1}$ State Key Laboratory of Automotive Safety and Energy, Tsinghua University, Beijing 100084, China \\ ${ }^{2}$ Tsinghua Automotive Strategy Research Institute, Tsinghua University, Beijing 100084, China \\ Correspondence should be addressed to Fuquan Zhao; zhaofuquan@tsinghua.edu.cn
}

Received 14 March 2019; Revised 13 June 2019; Accepted 24 June 2019; Published 24 July 2019

Academic Editor: Stefano de Luca

Copyright (c) 2019 Zongwei Liu et al. This is an open access article distributed under the Creative Commons Attribution License, which permits unrestricted use, distribution, and reproduction in any medium, provided the original work is properly cited.

\begin{abstract}
The development of intelligent vehicle will provide the Chinese automotive industry with a strategic opportunity for transformation and upgrading. Vehicular intelligence provides new solutions for energy conservation and emissions mitigation. However, the process of vehicular intelligence is progressive. The saving of energy consumption depends on the high smart car market penetration rate. But one thing that can be confirmed is that intelligent vehicles are equipped with advanced sensors, controllers, and actuators, in combination with connecting communication technologies compared with conventional vehicles, for which the energy consumption of the vehicle will definitely increase. In this study, vehicle fuel consumption cost at different levels of intelligence is calculated, considering the energy consumption of hardware used for automation and connecting functions, the energy consumption cost generated by the quality of the hardware, and the wind resistance. The results reveal that the energy consumption per 100 kilometers of an intelligent vehicle ranges from $0.78 \mathrm{~L}$ to $1.86 \mathrm{~L}$, more than traditional vehicle. The energy consumption cost of automation functions is much higher than that of the connecting functions. Computing platform performance, connection strength, and radar performance are the three main factors that affect energy consumption cost. Based on the analysis, the high energy consumption cost of vehicular intelligence has a profound impact on choosing power platform.
\end{abstract}

\section{Introduction}

As a major industry for energy consumption and greenhouse gas emissions, the automotive industry faces severe emission reduction challenges, and the process of low energy consumption and low carbonization is imminent [1]. The automotive industry is currently in a special historical period. Energy consumption, environmental pollution, traffic congestion, traffic accidents, and other issues have brought enormous challenges and pressures to the industry, and this pressure is particularly prominent in China [2]. With economic development, travel demands and logistic demands will continue to rise in China. To solve the associated problems, policies related to electric vehicle (EV) promotion and fuel economy regulations are being adopted by the state government [3]. According to the "Made in China 2025" strategy, intelligent vehicles, energy-saving vehicles, and new energy vehicles are the three major development priorities in the automotive area. The intelligent vehicle represents the commanding heights of prospective automotive technology, development trend, and industrial upgrading [4]. The automobile industry is characterized by large scale, strong driving effect, many fields involved, and a wide range of influences [5]. Therefore, the all-round changes in the automotive industry have given birth to historical opportunities [6].

The development of intelligent vehicles can significantly reduce energy consumption and greenhouse gas emissions was confirmed by many studies. Embedding algorithms for speed advice and economic driving advice in vehicle control systems can improve vehicle ride comfort and energy consumption [7]. Servin and Tielert discussed the effects of intelligent speed control and vehicle-traffic signal interaction on fuel consumption and emissions, respectively [8,9]. Adding vehicle fuel economy optimization algorithm to intelligent traffic light control can improve traffic efficiency and reduce vehicle fuel consumption [10]. From the perspective of roadcoordinated control technology, Lee evaluated traffic efficiency and safety improvements after developing self-driving 


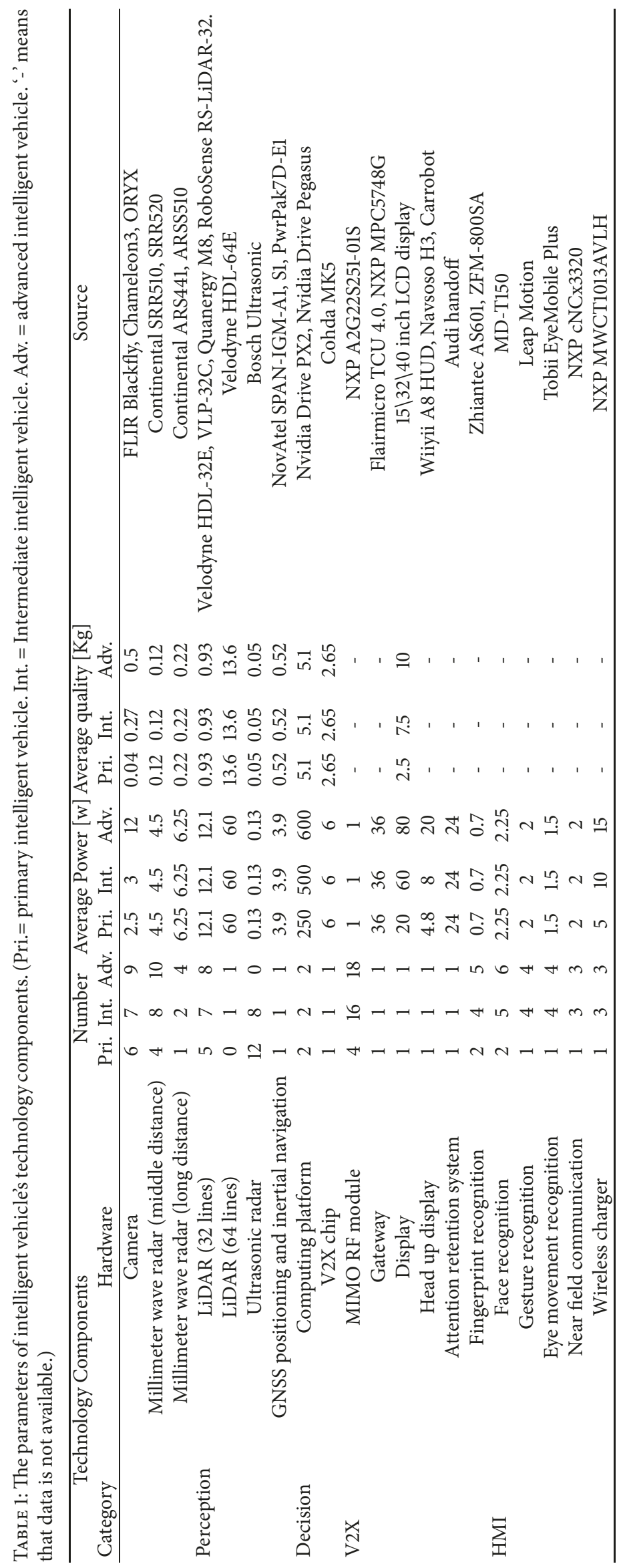




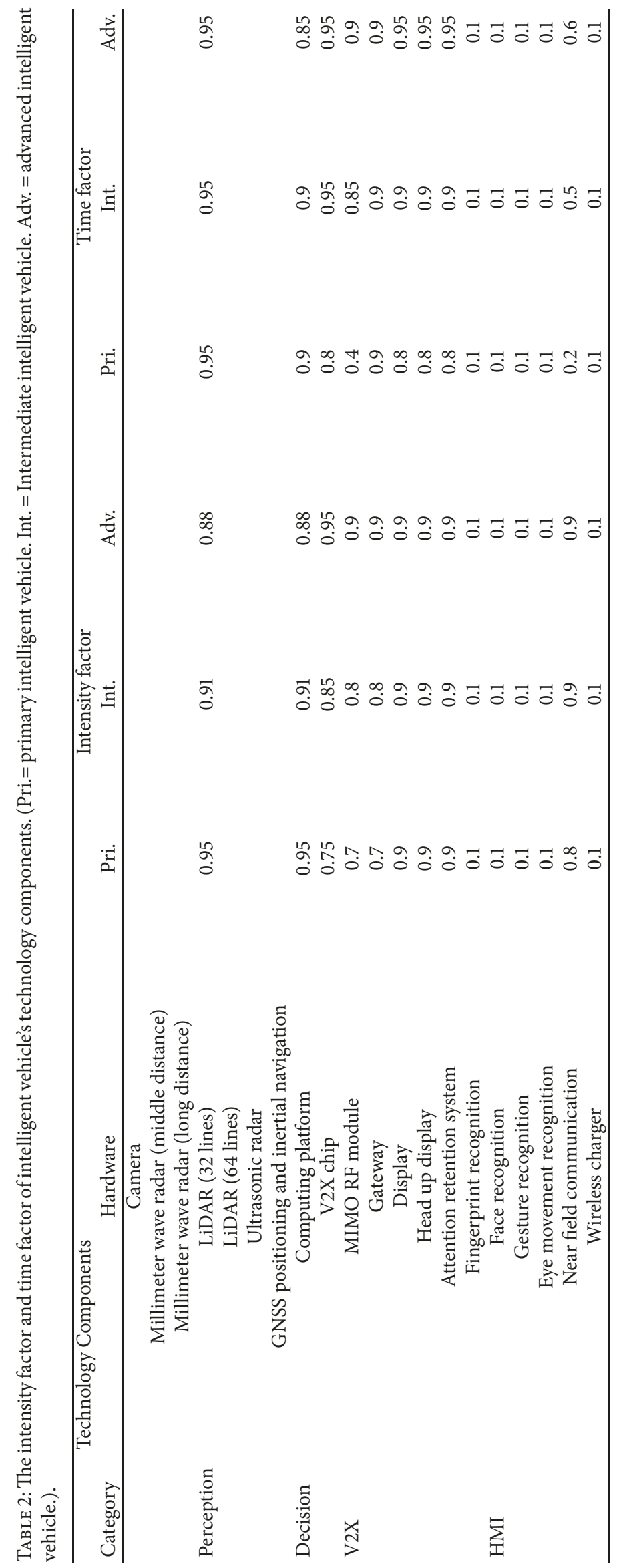


car [11]. Morris evaluated the impact of vehicle intelligence on real-time traffic efficiency. Based on this, the indirect impact mechanism of fuel consumption optimization due to traffic efficiency improvement was discussed [12]. The green wave band coordinated control intelligent technology can reduce the PM2.5 index in the environment along the road [13]. Yunpeng Wang verified the change of vehicle exhaust emissions under different standard driving speeds through the means of real road test [14]. Vehicle-to-vehicle communications report published by Harding J provided an overview of the development status of the V2X, from the perspective of technical application, facility construction, public acceptance, and regulatory rationality. And the costbenefit assessment of the impact of intelligent vehicles on fuel economy and traffic accidents was given in the report [15]. Maccubbin pointed out that intelligent safety assistance technology not only helps reduce accident rates, but also indirectly improves fuel consumption and traffic efficiency and reduces production costs and travel satisfaction [16].

In general, the existing research mainly focuses on the qualitative analysis of the macro intelligent traffic perspective, and the quantitative evaluation based on the individual vehicle intelligence is still rare. On the other hand, most of the evaluation dimensions of existing research are the positive benefits brought by the high intelligent vehicle market penetration rate, which is a synergistic benefit that relies heavily on high market penetration. But the number of intelligent vehicles is slowly increasing. In 2025, the market penetration rate of intelligent vehicles in new cars is $50 \%$ [17]. The proportion of intelligent vehicle in car ownership is relatively small, and group benefits are difficult to come by.

Compared with the traditional vehicle, the intelligent vehicle is equipped with various types of sensing hardware and decision hardware [19]. One thing that can be sure is that the intelligent vehicle will have a significant increase in driving energy consumption. The evaluation of such negative effects is still rare, and the relevant gaps need to be filled.

According to the requirements of the Medium and LongTerm Development Plan of the Automobile Industry, the average fuel consumption of new vehicles in 2020 needs to be reduced to 5.0 liters/100 kilometers, and 4.0 liters/100 kilometers in 2025[17]. Research on the promotion of new energy vehicles, especially electric vehicles, shows that the most effective way to increase the market share of new energy vehicles is to alleviate the "mileage anxiety" in the use of consumers [18]. The additional energy consumption generated by the intelligentization of automobiles will increase the difficulty of compliance with corporate regulations, shorten the cruising range of electric vehicles, and increase the cost of consumer use. Therefore, quantitative research on the energy consumption cost of Vehicular intelligentization and its key influencing factors will provide important support for the sustainable development of smart cars.

With the aim of filling such a gap, the fuel consumption cost per 100 kilometers of hardware mounted on the intelligent vehicle is estimated. Considering the possible technical routes, the energy consumption cost of the combination of different automation levels and connecting levels is evaluated. Moreover, multiple factors potentially influence the fuel consumption cost. Therefore, the key hardware that affects energy consumption is identified and a single factor analysis is also conducted in this study.

This paper is organized as follows. The next section describes the research method and data. Following that, the result of fuel consumption cost per 100 kilometers under different intelligent levels was given. The subsequent section proposed the policy implications for vehicle power platform. The final section provided the conclusive remarks.

\section{Materials and Methods}

This section describes the method and data in the study. In Section 2.1, the definition of vehicle Intelligence in the study is explained. Section 2.2 discusses the method in calculating energy consumption cost of vehicle intelligence. Following that, a brief description of data used are is given.

2.1. The Definition of the Intelligent Vehicle. Intelligentization is not the same as automation and connecting, but the result of the integration of automation and connecting. According to 'the Road Map of Energy Conservation and New Energy Vehicle Technology', the connecting capability is divided into three levels based on the amount of information transmitted over the network. L1 is connected auxiliary information interaction, L2 connected collaborative sensing, and L3 connected collaborative decision and control. The Society of Automotive Engineers (SAE) classifies the automated driving into six levels including No Driving Automation, Driver Assistance (DA), Partial Driving Automation (PA), Conditional Driving Automation (CA), High Driving Automation (HA), and Full Driving Automation (FA) [19]. Only basic autopilot capability and basic connecting capabilities are available, so DA and PA are not discussed in this article.

In this paper, the intelligent level is defined by the combination of the automation level and the connecting level, which is shown in Figure 1. A conditional driving automatic car with connected auxiliary information interaction is defined as a primary intelligent car. A high driving automatic car with connected collaborative sensing is defined as an intermediate intelligent car. A full driving automatic car with connected collaborative decision and control is defined as an advanced intelligent car.

\subsection{Energy Consumption Cost}

2.2.1. Source of Energy Consumption Cost. In order to identify the source of energy costs, it is necessary to define the definition of energy costs. The energy consumption cost refers to the increased energy consumption of intelligent vehicles traveling $100 \mathrm{~km}$ under NEDC conditions. NEDC is the driving condition in the fuel consumption measurement standard adopted by China's current light-duty vehicles. The $11 \mathrm{~km}$ full-length driving cycle takes 1180s. The average speed is $33.68 \mathrm{~km} / \mathrm{h}$ [20]. Therefore, this paper assumes that it takes 2.97 hours for the test intelligent vehicle to travel $100 \mathrm{~km}$, and it takes 0.33 hour to find the parking space. 


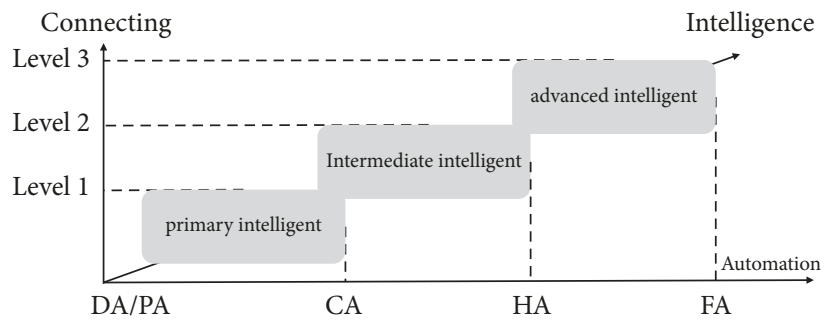

FIgURE 1: The definition of the intelligent vehicle.

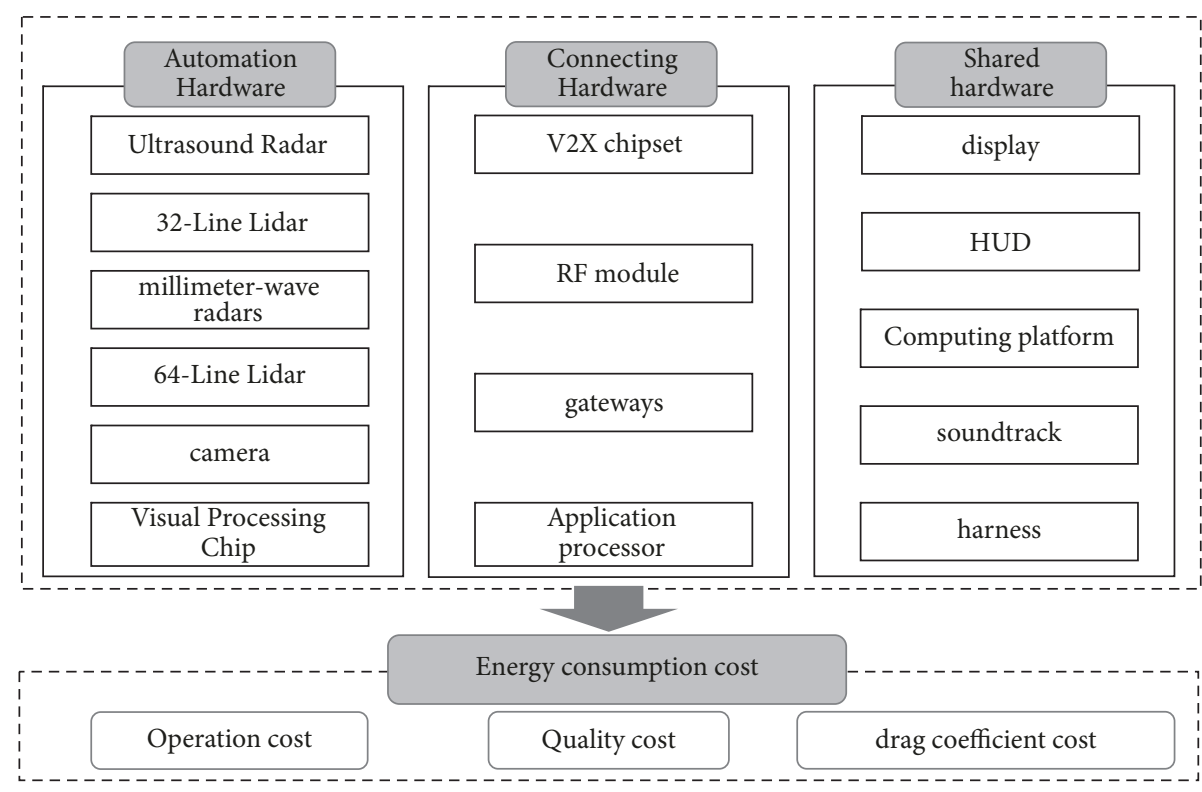

FIgURE 2: The energy consumption cost evaluation model.

The three major types of energy consumption costs due to assembly hardware are considered. The first part is the operating energy consumption of automation and connecting hardware. Different intelligent level vehicles are equipped with different numbers of hardware. The energy consumption of a single hardware also changes with the level of intelligence and the intensity of work. The second part is the energy consumption generated by the quality of the hardware. The third part is the energy consumption caused by the increase in the drag coefficient. The layout of the various types of sensors increases the drag coefficient of the vehicle. The energy cost calculation model of this study is shown in Figure 2.

2.2.2. Unit of Energy Consumption Cost. The energy consumption unit of electric vehicles is $\mathrm{kwh} / 100 \mathrm{~km}$, and $\mathrm{L} / 100 \mathrm{~km}$ of fuel vehicles. In order to make the energy consumption cost more intuitive, the unit of energy consumption cost in this paper is $\mathrm{L} / 100 \mathrm{~km}$. However, the energy consumption unit of the automation and connecting hardware is $\mathrm{kwh} / 100 \mathrm{~km}$, and the heat equivalent is used to convert the electric energy consumption into fuel consumption. The calorific value of gasoline is $4.6 \times 10^{\wedge} 7 \mathrm{~J} / \mathrm{Kg}$, and the density of gasoline is $0.7 \mathrm{Kg} / \mathrm{L}$. The corresponding calorific value of $1 \mathrm{kwh}$ is $3.6 \times 10 \wedge 6$ joules. Assume that the average thermal efficiency of a gasoline engine is $30 \%$. Based on this assumption, $1 \mathrm{~L}$ of gasoline can be calculated to produce $2.68 \mathrm{kwh}$ of electricity through the gasoline engine.

$$
1 \mathrm{~L} \times \frac{0.7 \mathrm{~kg}}{\mathrm{~L}} \times 4.6 \times 10^{7} \times 30 \% \div 3.6 \times 10^{6}=2.68 \mathrm{kwh}
$$

2.2.3. Hardware Operation Energy Consumption. The hardware operation energy consumption is divided into two parts: the automatic function hardware operation energy consumption and the connection function hardware operation energy consumption.

The automatic function hardware operation energy consumption under different intelligent levels is determined by the number of hardware, rated power, intensity factor, time factor, and driving time. The hardware related to automation functions is a set of automation hardware and common hardware such as laser radar and computing platform. The intensity factor is used to indicate that the working strength of each hardware is inconsistent during driving, and the same for time factor. 


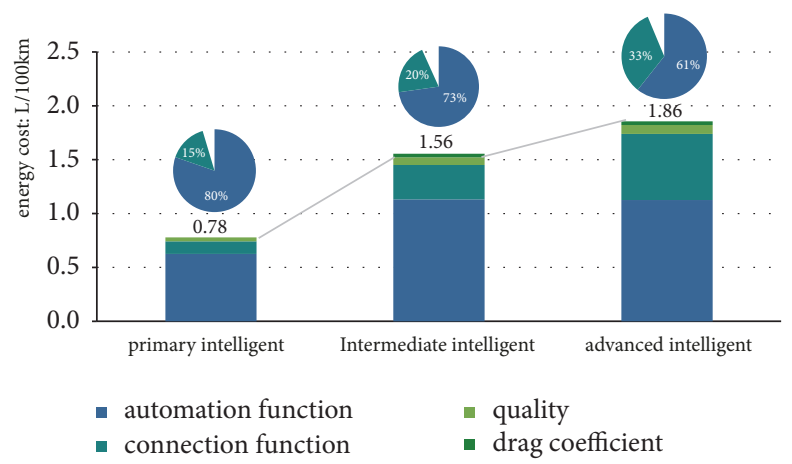

FIGURE 3: Vehicle fuel consumption cost at different levels of intelligence.

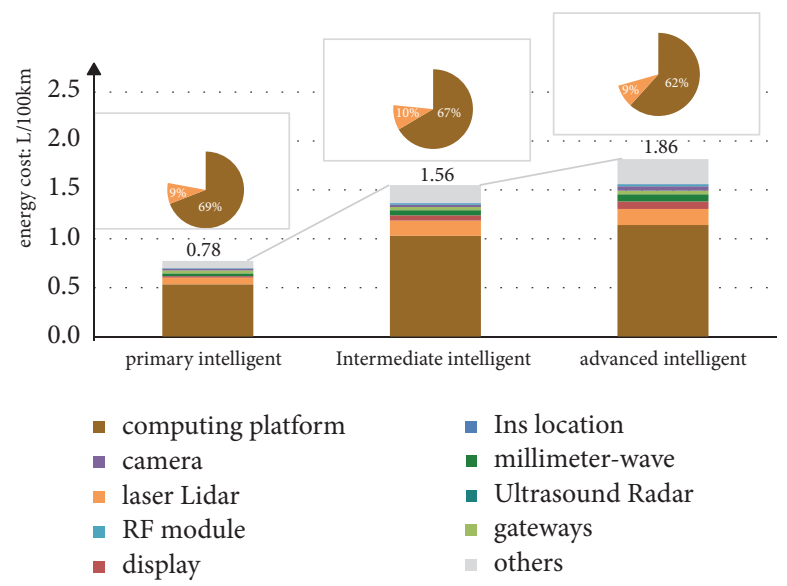

FIGURE 4: Fuel consumption components of various hardware at different levels of intelligence.

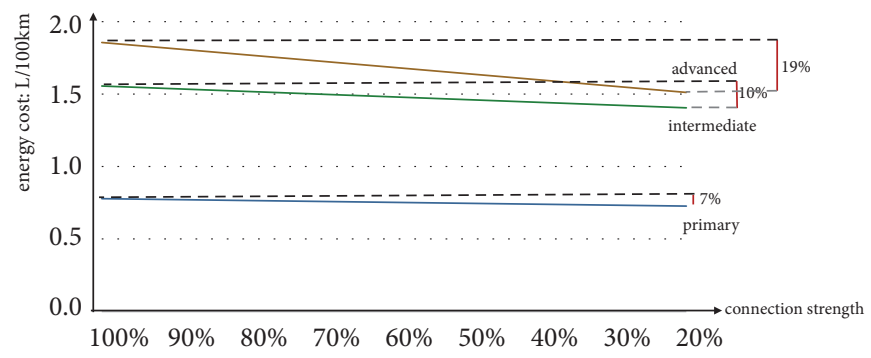

FIGURE 5: Fuel consumption cost of intelligent vehicle under different connection strength.

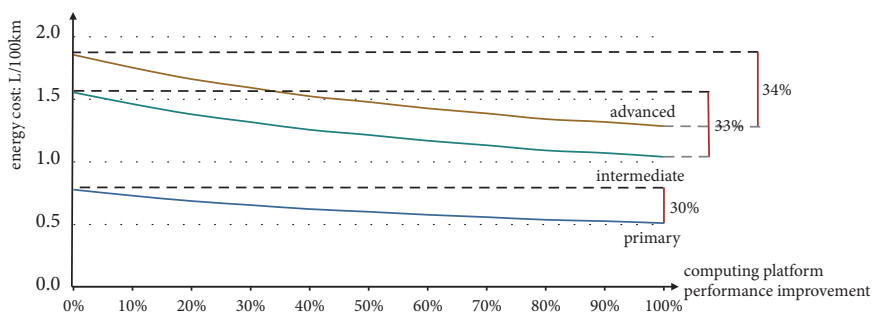

FIGURE 6: Fuel consumption cost of intelligent vehicle under computing platform performance improvement. 


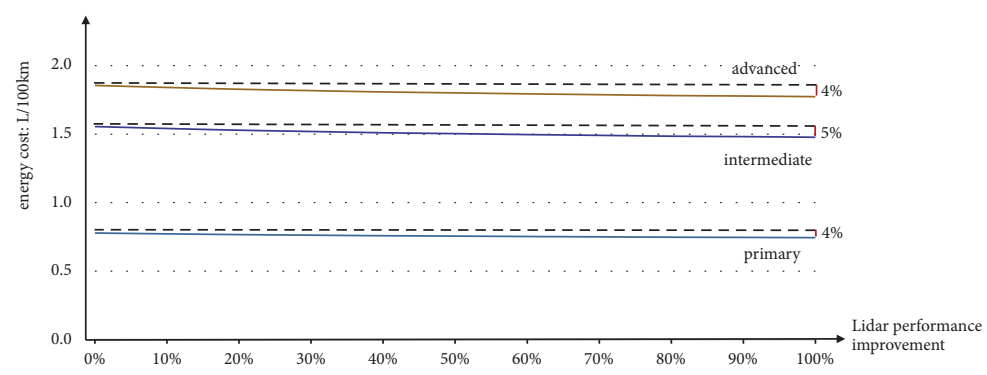

FIGURE 7: Fuel consumption cost of intelligent vehicle under LiDAR performance improvement.
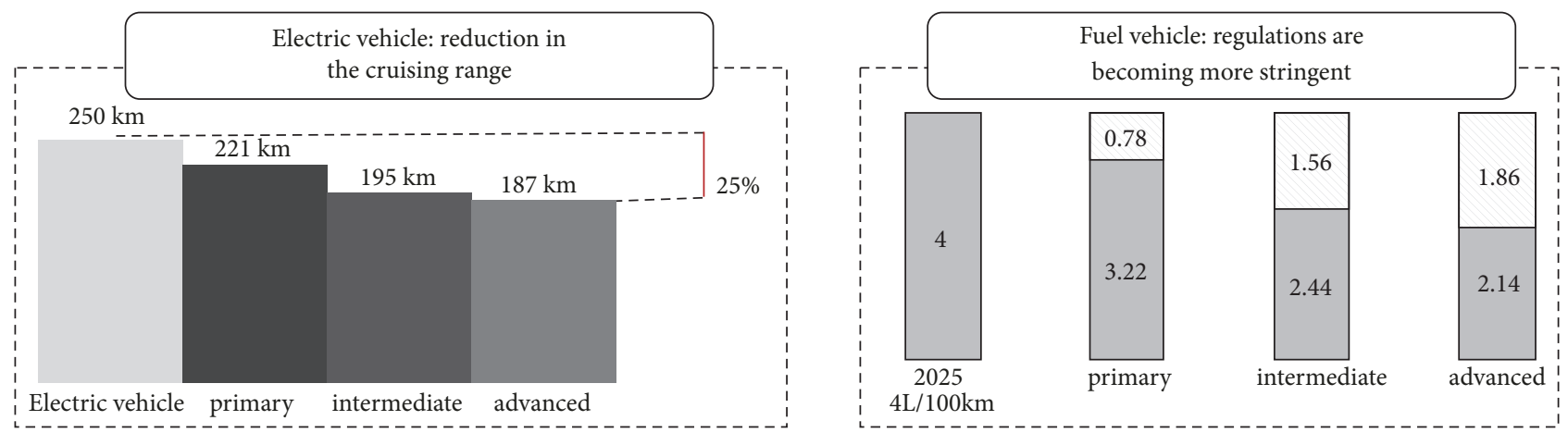

FIGURE 8: The impact of intelligent car on the fuel consumption of fuel vehicles and the cruising range of electric vehicles.

The automatic function hardware operation energy consumption is calculated through Eq. (2).

$$
\begin{aligned}
E C C_{A L}= & \sum_{k=0}^{1} \sum_{i=1}^{n}\left(\text { num }_{A i L} \times P_{A i L} \times I F a c_{A i L} \times T_{F a c}^{A i L}\right) \\
& \times t_{k} \times \text { Con }
\end{aligned}
$$

where $E C C_{A L}$ is the hardware operation energy consumption cost of the automation function of the intelligent car $(\mathrm{L} / 100 \mathrm{~km}) ; \mathrm{k}$ indicates whether there is a driver in the car; num $_{A i L}$ is the number of type i automation hardware equipped with the intelligent cars; $P_{A i L}$ is the rated power of the relevant hardware $(\mathrm{kw}) ; I F a c_{A i L}$ is the intensity factor of the relevant hardware; $T F a c_{A i L}$ is the time factor of the relevant hardware; $t_{k}$ is the travel time of the vehicle in the $\mathrm{k}$ state (h); Con is the Unit conversion factor.

The connection function hardware operation energy consumption under different intelligent levels is also determined by the number of hardware, rated power, intensity factor, time factor, and driving time. The hardware associated with the connection function is a series of connected hardware and common hardware, such as V2X chip, MIMO RF module, gateway and computing platform. The connection function hardware operation energy consumption is calculated through Eq. (3).

$$
\begin{aligned}
& E C C_{C L}=\sum_{k=0}^{1} \sum_{i=1}^{m}\left(\operatorname{num}_{C i L} \times P_{C i L} \times I F a c_{C i L} \times T F F a c_{C i L}\right) \\
& \times t_{k} \times \text { Con }
\end{aligned}
$$

where $E C C_{C L}$ is the hardware operation energy consumption cost of the connection function of the intelligent car $(\mathrm{L} / 100 \mathrm{~km}) ; \mathrm{k}$ indicates whether there is a driver in the car; num $_{\text {CiL }}$ is the number of connection hardware equipped with the intelligent cars; $P_{C i L}$ is the rated power of the relevant hardware (kw); $I F a c_{C i L}$ is the intensity factor of the relevant hardware; $\mathrm{TFac}_{\mathrm{CiL}}$ is the time factor of the relevant hardware; $t_{k}$ is the travel time of the vehicle in the $\mathrm{k}$ state $(\mathrm{h})$; Con is the Unit conversion factor.

The computing platform is involved in equations (2) and (3). Because the sensing hardware transmits the information needed for automation to the computing platform for processing and decision making, as well as the connecting information. Therefore, it is necessary to reasonably distinguish the energy consumption of the automation and connecting functions in the computing platform. The principle of differentiation is based on the amount of data. The amount of LiDAR transmits to the computing platform is $1 \mathrm{M} / \mathrm{s}$. The amount of connection information transferred to the computing platform is related to the connecting capability.

2.2.4. Hardware Quality. Energy consumption generated by the quality of the hardware is determined by travel speed, rolling resistance coefficient, increased hardware quality, and travel time. When driving on asphalt road at a speed of $33.68 \mathrm{~km} / \mathrm{h}$, the rolling resistance coefficient is 0.0157 [21].

The energy consumption generated by the quality is calculated through Eq. (4).

$$
E C C_{\mathrm{Q} L}=\sum_{i=1}^{n} m_{i L} \times 9.8 \times f \times v \times t \times C o n \div 1000
$$


where $E C C_{\mathrm{QL}}$ is the energy consumption generated by the hardware quality $(\mathrm{L} / 100 \mathrm{~km}) ; m_{i L}$ is the hardware quality $(\mathrm{kg})$; $f$ is the rolling resistance coefficient of asphalt pavement; $v$ is the travel speed $(\mathrm{m} / \mathrm{s}) ; t$ is the travel time $(\mathrm{h})$.

2.2.5. Wind Resistance. Externally mounted hardware on the roof rack will increase the drag coefficient. According to the evaluation, the roof rack increases the energy consumption of the vehicle by $0.05 \%$ [22].

The energy consumption caused by the increase in the drag coefficient is calculated through Eq. (5).

$$
\begin{aligned}
E C C_{D L}= & 0.005 \\
& \times\left(E C C_{A L}+E C C_{C L}+E C C_{Q L}+E C_{B}\right)
\end{aligned}
$$

where $E C C_{D L}$ is the energy consumption caused by the increase in the drag coefficient $(\mathrm{L} / 100 \mathrm{~km}) ; E C C_{A L}$ is the hardware operation energy consumption cost of the automation function of the intelligent car $(\mathrm{L} / 100 \mathrm{~km}) ; E C C_{C L}$ is the hardware operation energy consumption cost of the connection function of the intelligent car $(\mathrm{L} / 100 \mathrm{~km}) ; E C C_{\mathrm{Q} L}$ is the energy consumption generated by the hardware quality $(\mathrm{L} / 100 \mathrm{~km}) ; E C_{B}$ is the energy consumption of the traditional car, which is assumed to be $13.6 \mathrm{kwh} / 100 \mathrm{~km}$.

2.3. Data Description. According to the calculation model, the required data is the list of the hardware, including types, number, average rated power, and average quality. For example, for computing platform, we selected the Nvidia Drive PX2 for the primary intelligent vehicle and the Nvidia Drive Pegasus for the Intermediate intelligent vehicle. The average power is $500 \mathrm{w}$ and $600 \mathrm{w}$, while the quality is both $5.1 \mathrm{~kg}$. It is important to note that the computing platform is a key component. In order to ensure the safety of intelligent vehicles, the redundancy of the computing platform needs to be considered. So, it is assumed that the intelligent vehicle was equipped with two computing platforms. And, for the 32-line LiDAR, we selected Velodyne HDL-32E, Velodyne VLP-32C, Quanergy M8, and RoboSense RS-LiDAR-32. They have an average quality of $0.93 \mathrm{~kg}$ and an average power of $12.1 \mathrm{~W}$. And for millimeter wave radar (middle distance), Continental SRR510 and Continental SRR520 were selected. They have an average quality of $0.12 \mathrm{~kg}$ and an average power of 4.5W. More details are shown in Table 1.

According to the definition of intelligent vehicle in the Section 2.1, only a few primary intelligent vehicles are being tested. And the hardware configuration of intelligent vehicles that developed by various companies is different. So the number of each hardware is not clear yet. Even more difficult is that current hardware solutions are still uncertain and most companies are not actively sharing data that is considered to be trade secrets. In the future, hardware solutions may also be completely changed as technology advances. For example, Tesla has always insisted that automatic driving does not require a laser radar. Because of those uncertainties, we have found some representative companies' products for analysis, sorted out more detailed and specific data, and gave a quantitative hypothesis of the number of each hardware. The Cruise
AV is equipped with 5 LiDARs, 16 cameras, and 21 radars [23]. And the number of the hardware equipped on the Ford and Waymo's self-driving vehicles has not been announced in the safety report $[24,25]$. By investigating a large number of professional literature, product manuals, research reports, and interviewing relevant experts, the number of hardware is assumed, as shown in the Table 1 . The intensity factor of the relevant hardware and the time factor of the relevant hardware are defined by the relevant expert. Relatively, its value will be a little subjective. But in order to show more complete and detailed calculation process, intensity factor and time factor are shown in Table 2.

\section{Results and Discussion}

This section presents the results and discussions in the study. Section 3.1 shows the fuel consumption cost at different levels of intelligence. Section 3.2 shows the Fuel consumption components of various hardware at different levels of intelligence, identifying the key hardware that affects the fuel consumption cost. After that, Section 3.3 conducts a singlefactor analysis to compare the influencing factors.

3.1. Fuel Consumption Cost. According to the data assumption and model above, the fuel consumption cost at different levels of intelligence is shown in Figure 3, considering the energy consumption caused by the additional intelligent hardware operation, quality and drag coefficient. Automotive intelligence dramatically increases the fuel consumption. Compared with traditional cars, the fuel consumption of a primary intelligent car increases by $0.78 \mathrm{~L} / 100 \mathrm{~km}$. The fuel consumption of an advanced intelligent car increases by $1.86 \mathrm{~L} / 100 \mathrm{~km}$. The cost of fuel consumption increases as the level of intelligence increases. From the composition of the energy consumption cost of the intermediate intelligent car, it can be found that the automation function has the highest fuel consumption ratio, up to $80 \%$. And the connection function is second, accounting for $23 \%$. In contrast, energy consumption cost generated by the quality and increased drag coefficient is small.

3.2. The Key Hardware. The energy consumption cost of automotive intelligence is generated by new hardware. According to the number, quality, power, intensity factor, and time factor, the energy consumption cost ratio of each hardware under different intelligence levels is calculated, as shown in Figure 4. Computing platforms, LiDAR, millimeterwave radars, displays, gateways, and cameras are all relatively high-energy hardware. Among them, the computing platform has the highest energy consumption, up to $69 \%$ in the primary intelligent car. This is due to the fact that, regardless of the automation function or the connecting function, all data processing and decision-making are performed by the computing platform. As the level of intelligence increases, the amount of data increases. The increase in the amount of data that needs to be processed makes the computing platform consume more energy. Secondly, the energy consumption cost of laser radar accounts for $10 \%$ of the intermediate 
intelligent car. This is because the number of laser radars required for autonomous driving is high. For example, the number of different types of radars equipped in the advanced intelligence class is up to 24 . In general, the most critical hardware in the energy cost ratio is the computing platform and LiDAR.

3.3. Single-Factor Analysis. Multiple factors potentially influence the assessment of energy consumption cost. In order to evaluate the impact of various influencing factors on energy costs, a single factor analysis of key influencing factors is performed in the following sections. Influencing factors including connection strength, computing platform performance, and LiDAR performance are considered.

3.3.1. Connection Strength. Connection time and data transmission volume are key parameters that decide the effect in reducing fuel consumption cost of intelligent cars. Connection strength is defined as the multiplier of on-line time and data transmission volume. Connection strength benchmark (100\%) is assumed to be $95 \%$ connection time and rated power operation. In order to meet the most basic functional requirements, the connection strength is required to be no less than $20 \%$. Fuel consumption costs of intelligent vehicle under different connection strength are compared in Figure 5.

The connection strength has relatively large influence on the fuel consumption cost of intelligent vehicles. When the connection strength is changed from $100 \%$ to $20 \%$, the fuel consumption costs are influenced by $7 \%, 10 \%$, and $19 \%$ in primary, intermediate, and advanced intelligent cars. Connection strength has the greatest influence on advanced intelligent vehicle.

Since the connection strength is directly related to the user experience, the enterprise cannot technically limit the connection strength and can only guide the consumers to use the connecting function less.

3.3.2. Computing Platform. As explained in Section 3.2, the hardware that accounts for the highest proportion of energy consumption costs for intelligent cars is the computing platform. The computational speed and rated power of the computing platform are key parameters that determine the energy cost of computing platform. Computing platform performance improvement is defined as the ratio of computational speed progression to rated power advancement. For example, if the computing platform's rated power is fixed and the calculation speed is doubled, the performance is increased by $100 \%$. Or the computing platform's calculation speed is fixed, but the rated power is reduced by half, and the performance is increased by $100 \%$. The energy consumption cost of different levels of intelligent cars under different computing platform performance is shown in Figure 6.

Computing platform performance has a significant impact on the energy cost of the intelligent car. When the performance is doubled compared with the current situation, the energy consumption cost of the primary, intermediate, and advanced intelligent car is reduced by $30 \%, 33 \%$, and $34 \%$, respectively. The impact on a higher level of intelligence is greater. It can be concluded that a low-energy consumption computing platform is an important core technology for the intelligent car.

3.3.3. LiDAR. As explained in Section 3.2, the hardware that accounts for the second highest proportion of energy consumption costs for the intelligent car is the LiDAR. The sensing capability and rated power of the LiDAR are key parameters that determine the energy cost of the LiDAR. LiDAR performance improvement is defined as the ratio of the sensing capability progression to the rated power advancement, which is as similar as the computing platform. The energy consumption cost of different levels of intelligent cars under different LiDAR performance is shown in Figure 7.

Compared with the connection strength and the performance of the computing platform, LiDAR performance has little impact on the energy consumption cost of the intelligent car. Even if the performance of the LiDAR is doubled, it can only reduce the energy consumption cost by $5 \%$. This shows that if only one component just like LiDAR is improved, the energy consumption cost of the car reduction is small. In the future, the improvement of energy consumption of the intelligent car should emphasize the overall optimization.

\section{Policy Implications}

The average fuel consumption of new vehicles in 2020 needs to be reduced to 5.0 liters/100 kilometers, and 4.0 liters/100 kilometers in 2025 [17]. Fuel vehicle's fuel consumption regulations are becoming more stringent. Based on the $4 \mathrm{~L} / 100 \mathrm{~km}$ regulatory requirements in 2025 , the primary intelligent car only has an upper fuel consumption limit of $3.22 \mathrm{~L} / 100 \mathrm{~km}$ for driving for fuel vehicle. And the intelligent energy consumption cost of the advanced intelligent car is $1.56 \mathrm{~L} / 100 \mathrm{~km}$, leaving only the upper limit of $2.44 \mathrm{~L} / 100 \mathrm{~km}$ for driving. Intelligentization greatly increases the difficulty of the automotive products to meet regulatory requirements. The development of intelligent cars using fuel vehicle platforms will face the challenge of severe regulatory compliance.

Follow-up analysis is based on an electric car with a cruising range of $250 \mathrm{~km}$. As the total energy of the vehicle battery is fixed, supplying more energy to various type of hardware leads to a significant reduction in the cruising range of the electric vehicle. Based on equation (1), the intelligent fuel consumption cost was converted into electricity cost. The cruising range of advanced intelligent electric vehicles was reduced to $187 \mathrm{~km}$, as shown in Figure 8. A drop of up to 25\% is unacceptable to consumers. On the other hand, such a high energy cost means a significant increase in the cost of use. This leads consumers to think more about whether to buy an intelligent electric car.

The electrification of automobiles is the general trend. Fuel consumption regulations are becoming more stringent, and it is difficult to meet regulatory standards if batteries are not available when necessary. On the other hand, battery power can respond to intelligent demands while automatically driving as soon as possible. Therefore, it is necessary to assemble the battery in the car. 
However, it is not feasible to rely solely on the battery. Intelligent function will increase the load on the power battery. On the other hand, the internal combustion engine technology is relatively mature, and the cost of increasing the number of batteries is much higher than the cost of increasing the volume of the fuel tank.

Therefore, Range Electric Vehicle (REV) and Plug-in Hybrid Electric Vehicle (PHEV) will become a long-term choice, which is the combination of electricity-based energy consumption and oil-based energy storage.

\section{Conclusions}

There exist studies that focus on the energy impacts of intelligent vehicle. These studies discussed the energy savings of intelligent vehicles. However, few studies are conducted to evaluate negative impact of intelligent vehicles on energy consumption. As developing intelligent vehicles is the trend of the times and 50\% of the vehicles sold in China in 2025 are intelligent vehicles, a detailed study on negative impact on energy consumption is necessary. With the aim of filling such a gap, in this study, the fuel consumption cost per 100 kilometers of hardware mounted on the intelligent vehicle is estimated. Moreover, multiple factors potentially influence the fuel consumption cost. Therefore, the key hardware that affects energy consumption is identified and a single factor analysis is also conducted in this study.

In this study, vehicle fuel consumption costs at different levels of intelligence are calculated, considering the energy consumption of the automation function and connecting function, the energy consumption cost generated by the quality, and the wind resistance. Based on the research, vehicular intelligentization significantly increases driving fuel consumption. The energy consumption increments from primary to advanced are $0.78,1.56$, and $1.86 \mathrm{~L} / 100 \mathrm{~km}$, respectively. Among them, the automation function consumed the highest proportion, up to $80 \%$ in advanced intelligence scene. The connecting function consumed the second, up to $33 \%$ in advanced primary scene. The energy consumption cost generated by the quality and the wind resistance of hardware quality account for a relatively small proportion.

Single factor analysis is also conducted in this study. The most impressive influencing factors include connection strength, computing platform performance, and LiDAR performance. The results indicate that computing platform is the key hardware that has the greatest impact on energy consumption costs. The significant increase in computing platform performance can reduce the fuel consumption cost of primary, middle, and advanced intelligence by $30 \%, 33 \%$, and $34 \%$, respectively. When the connection strength is changed from $100 \%$ to $20 \%$, the fuel consumption cost was influenced by $7 \%, 10 \%$, and $19 \%$. However, the significant increase in LiDAR performance can only reduce the fuel consumption cost by $5 \%$.

The suggestion for choosing power platform of intelligent vehicle is proposed in this study. Vehicular Intelligentization has become the trend of the times, which will change the choice of vehicle power platform and technical route for a long time. On the one hand, the cruising range of electric vehicles has been shortened. On the other hand, it is more difficult to meet the fuel consumption regulations of fuel vehicles. Therefore, REV and Plug-in Hybrid Electric Vehicle, the combination of electricity-based energy consumption and oil-based energy storage, will become a long-term choice.

This study still has its limitations. Firstly, the operating intensity factor of various types of hardware is assumed in this study. This assumption is due to a lack of reliable data. Secondly, the energy consumption cost of redundancy in steering and braking systems is not considered, which may increase the energy consumption cost in the future. Thirdly, only the negative impact of vehicular intelligentization on energy consumption is discussed. The results cannot show the complete impact of vehicular intelligentization on energy because this research focuses on the negative impact of the intelligent vehicle on energy consumption.

\section{Data Availability}

The data used to support the findings of this study are available from the corresponding author upon request.

\section{Conflicts of Interest}

The authors declare that there are no conflicts of interest regarding the publication of this paper.

\section{Acknowledgments}

This study is sponsored by the National Natural Science Foundation of China (U1764265, 71774100, 71403142, 71690241). This study is also sponsored by the Saudi Arabian Oil Company (20173000217).

\section{References}

[1] F. Zhao, F. Liu, and Z. Liu, "Research on china's automobile industry low carbonization evaluation index system," Engineering Science, vol. 20, no. 1, 2018.

[2] F. Zhao, R. Su, and Z. Liu, Turning China into a Stronger Automotive Country: A Practice, China Machine Press, Beijing, China, 2017.

[3] F. Zhao, F. Liu, Z. Liu, and H. Hao, “The correlated impacts of fuel consumption improvements and vehicle electrification on vehicle greenhouse gas emissions in China," Journal of Cleaner Production, vol. 207, pp. 702-716, 2019.

[4] China Daily, Made in China 2025 Strategy for Auto Industry, 2015, http://english.gov.cn/policies/infographics/2015/06/02/ content_281475119391820.htm.

[5] F. Zhao and Z. Liu, "Research on china's manufacturing transformation strategy under the industry 4.0 wave," Forum on Science and Technology in China, vol. 1, pp. 58-68, 2016.

[6] F. Zhao, "Strategic thinking on the opportunity of the automobile industry subversion period and the countermeasures of traditional vehicle enterprises," Modern Components, vol. 12, pp. 16-18, 2016.

[7] J. D. Vreeswijk, M. K. M. Mahmod, and B. V. Arem, "Energy efficient traffic management and control-the eCoMove approach 
and expected benefits," in Proceedings of the 13th International IEEE Conference on Intelligent Transportation Systems, ITSC 2010, pp. 955-961, 2010.

[8] O. Servin, K. Boriboonsomsin, and M. Barth, "An energy and emissions impact evaluation of intelligent speed adaptation," in Proceedings of the ITSC 2006: 2006 IEEE Intelligent Transportation Systems Conference, pp. 1257-1262, IEEE, Canada, September 2006.

[9] T. Tielert, M. Killat, H. Hartenstein, R. Luz, S. Hausberger, and T. Benz, "The impact of traffic-light-to-vehicle communication on fuel consumption and emissions," in Proceedings of the 2nd International Internet of Things Conference, IoT 2010, pp. 1-8, IEEE, Japan, December 2010.

[10] H. Rakha and R. K. Kamalanathsharma, "Eco-driving at signalized intersections using V2I communication," in Proceedings of the 14th IEEE International Intelligent Transportation Systems Conference (ITSC '11), pp. 341-346, IEEE, Washington, DC, USA, October 2011.

[11] J. Lee, B. Park, K. Malakorn, and J. So, "Sustainability assessments of cooperative vehicle intersection control at an urban corridor," Transportation research part c: emerging technologies, vol. 32, pp. 193-206, 2013.

[12] M. Ferreira and P. M. D’Orey, "On the impact of virtual traffic lights on carbon emissions mitigation," IEEE Transactions on Intelligent Transportation Systems, vol. 13, no. 1, pp. 284-295, 2012.

[13] X. Hu, W. Li, and G. Shen, "Analysis of the impact of the application of intelligent transportation system on PM2.5 in the environment along the road," Environmental Pollution \& Control, vol. 35, no. 5, pp. 45-48, 2013.

[14] Y. Wang, D. Guo, and H. Qi, "Impact of urban graded road vehicle speed on emissions," Journal of Harbin Institute of Technology, vol. 41, no. 7, pp. 110-114, 2009.

[15] J. Harding, G. Powell, and R. Yoon, Vehicle-to-Vehicle Communications: Readiness of V2V Technology for Application, 2014.

[16] P. R. Maccubbin, L. B. Staples, and M. R. Mercer, Intelligent Transportation Systems Benefits and Costs: 2003 Update, 2003.

[17] The Ministry of Industry and Information Technology, Medium and Long-Term Development Plan of the Automobile Industry, 2017, http://www.most.gov.cn/tztg/201705/t20170510_ 132694.htm.

[18] S. Su, J. Sun, and X. Lin, "Intelligent charging navigation for electric vehicles," in Proceedings of the CSEE, pp. 59-67, 2013.

[19] SAE International, SAE J3016(TM), 2018.

[20] Ministry of Ecology and Environment of the people's Republic of China, "GB 18352.5-2013. Limits and measurement methods for emissions from light-duty vehicles (CHINA)5[S]," 2013.

[21] Z. Yu, Automobile Theory [M], China Machine Press, Beijing, China, 2009.

[22] Y. Chen and A. Meier, "Fuel consumption impacts of auto roof racks," Energy Policy, vol. 92, pp. 325-333, 2016.

[23] Waymo, On the Road to Fully Self-driving: Waymo Safety Report, 2017, https://waymo.com/safety/.

[24] Ford, "A mattrt of trust: Ford's approach to developing self-driving vehicle," https://media.ford.com/content/dam/ fordmedia/pdf/Ford_AV_LLC_FINAL_HR_2.pdf.

[25] General Motors, 2018 Self-Driving safety report, January 18, Accessed, 2018, https://www.gm.com/our-stories/self-drivingcars.html. 


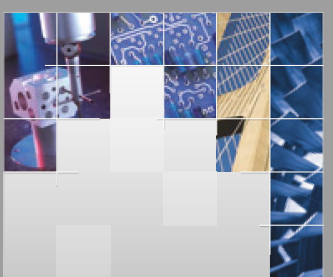

\section{Enfincering}
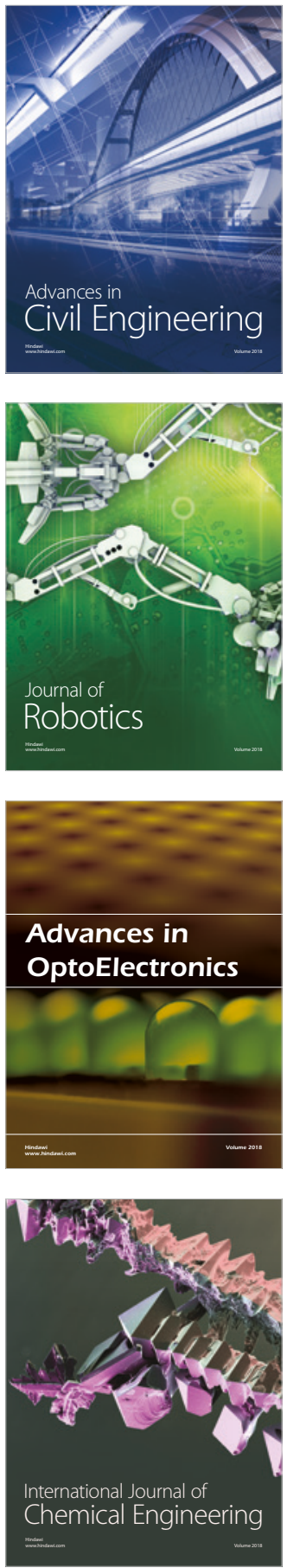

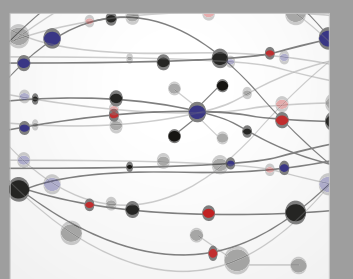

\section{Rotating \\ Machinery}

The Scientific World Journal

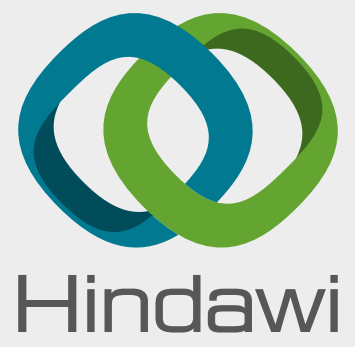

Submit your manuscripts at

www.hindawi.com
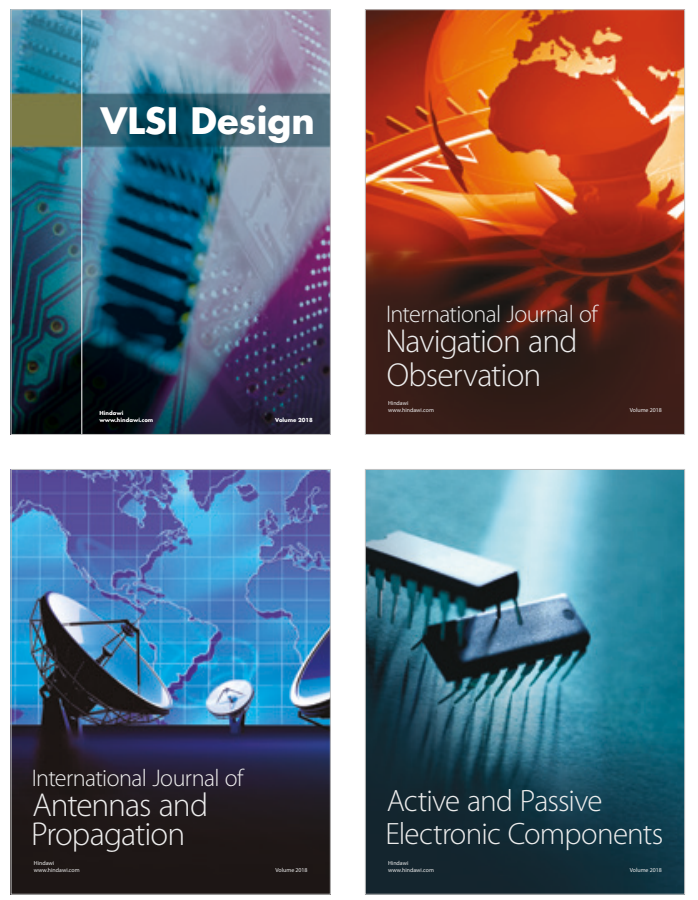
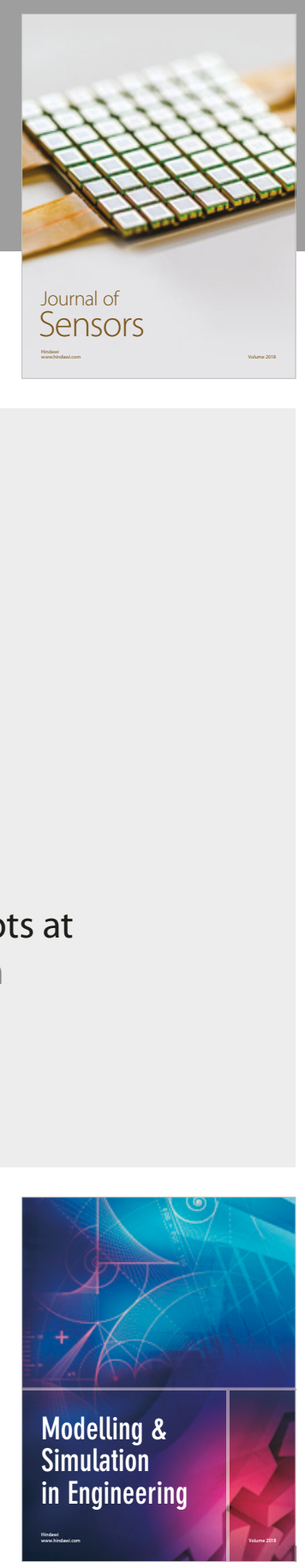

\section{Advances \\ Multimedia}
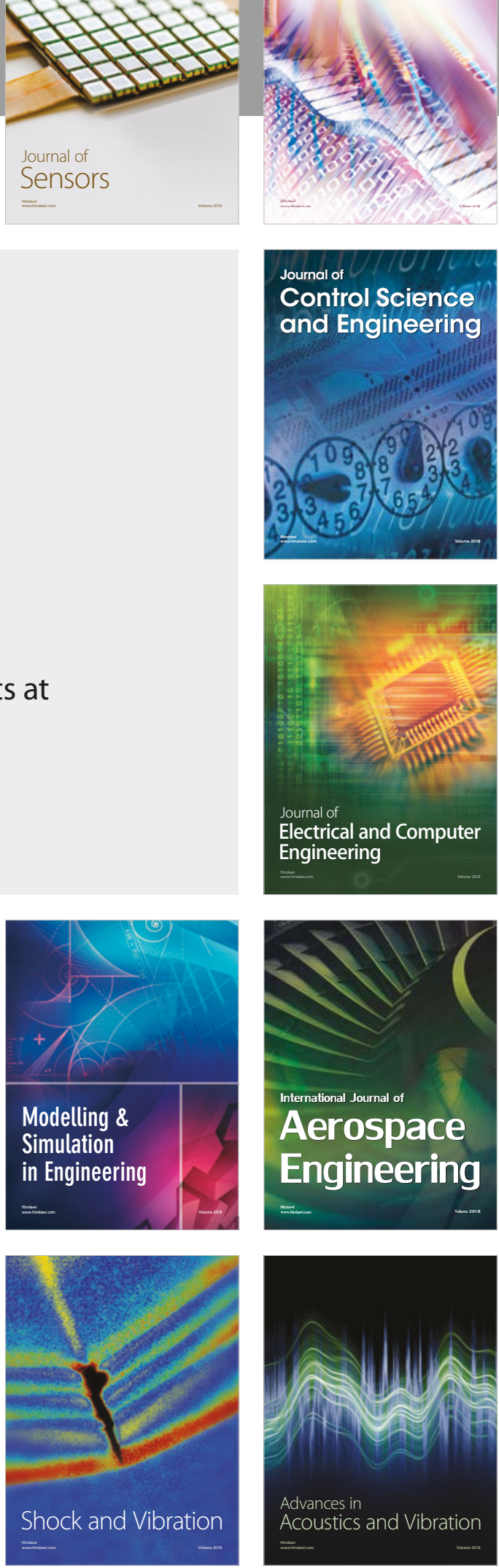